\title{
CORRELATION OF ANAEMIA WITH ATTENUATION VALUES ON UNENHANCED CT OF THORAX
}

\author{
Nandakishore G. Patili1, Chetan Mehta², Shreya Sehgal3, Juhi Patel ${ }^{4}$, Abhishek $S^{5}$
}

${ }^{1}$ Resident, Department of Radiodiagnosis, Government Medical College and SSG Hospital, Vadodara.

${ }^{2}$ Associate Professor, Department of Radiodiagnosis, Government Medical College and SSG Hospital, Vadodara.

${ }^{3}$ Resident, Department of Radiodiagnosis, Government Medical College and SSG Hospital, Vadodara.

${ }^{4}$ Resident, Department of Radiodiagnosis, Government Medical College and SSG Hospital, Vadodara.

${ }^{5}$ Resident, Department of Radiodiagnosis, Government Medical College and SSG Hospital, Vadodara.

\section{ABSTRACT}

\section{BACKGROUND}

Anaemia is one of the most common clinical conditions encountered in India. Our aim was to determine the importance of CT attenuation value in prediction of anaemia in unenhanced CT scan of thorax.

\section{MATERIALS AND METHODS}

A retrospective review of 70 patients who had haemoglobin levels obtained within 72 hours of an unenhanced CT thorax was done. Regions of interest were placed in the lumen of aorta at the region of bifurcation of main pulmonary artery. CT attenuation values were correlated with haemoglobin to determine the possible existence of any possible relationship.

\section{RESULTS}

The CT attenuation value of aorta was found to be more sensitive while the interventricular septum sign was found to be more specific in the detection of anaemia. A good correlation $(r=0.63)$ was observed between the aortic CT attenuation values and the haemoglobin levels in the study group.

\section{CONCLUSION}

The attenuation values of aorta, aortic ring sign and the interventricular septum sign on unenhanced CT scan of thorax constitute a practical adjunct in the assessment of anaemia. This rapid assessment technique for anaemia is a convenient means for narrowing possible diagnoses. Consent was taken from the Scientific and ethical review committee, Government Medical College and SSG Hospital, Vadodara.

\section{KEYWORDS}

Anaemia, CT Thorax, CT Attenuation Values, Haemoglobin.

HOW TO CITE THIS ARTICLE: Patil NG, Mehta C, Sehgal S, et al. Correlation of anaemia with attenuation values on unenhanced CT of thorax. J. Evolution Med. Dent. Sci. 2017;6(17):1309-1312, DOI: 10.14260/Jemds/2017/284

\section{BACKGROUND}

CT examination of the thorax and other parts of the body often produces many incidental findings.(1) Identifying such incidental findings which are clinically significant may impose significant impact on patient management and outcome.(2,3) The measurement of an attenuation value of an anatomical region at CT examination is common.

Patients under acute situations most often do not undergo complete blood cell count or other haematologic laboratory investigations at the time of imaging particularly in the setting of trauma. Of particular diagnostic significance is the haemoglobin level, a low value that can be associated with chest and abdominal trauma patients. Diagnosis of anaemia in unenhanced CT scans in emergency settings helps in favourable outcome to the patient.(4-7) The measured attenuation value is a reproducible physical density measurement, readily

Financial or Other, Competing Interest: None.

Submission 04-12-2016, Peer Review 12-02-2017,

Acceptance 18-02-2017, Published 27-02-2017.

Corresponding Author:

Dr. Nandakishore G. Patil,

Plot No. 4, Saptagiri,

Chidambarnagar Housing Society,

Gandhinagar, Dharwad-580004,

Karnataka.

E-mail: nandakishorepatil88@gmail.com

DOI: $10.14260 /$ jemds $/ 2017 / 284$ obtainable from a standard CT examination. Some investigators have previously reported the incidental detection of severe anaemia on unenhanced CT of the thorax. ${ }^{8-13)}$ It has been shown that relative hyperdense appearance of myocardium and interventricular septum has been a sign of anaemia.(14) Similarly, hyperattenuating aortic wall has also been considered to be a reliable sign in the diagnosis of anaemia. This approach, however, has been recently shown to be an operator-dependent process that may be subject to significant inter-observer variability.(15)

However, the above-mentioned signs are seen in other conditions also, like the storage disorders where the relative hyperdense appearance of interventricular septum and myocardium is seen.(16) The aortic ring sign is also a nonspecific sign which is seen in age related atherosclerotic wall thickening, mural plaques in various conditions like hypertension, infective states like chronic syphilis and inflammatory conditions like giant cell arteritis. Thus, the primary purpose of this study is to evaluate if, on an unenhanced CT, the attenuation of blood in the aorta correlates with haemoglobin levels and to evaluate the specificity of aortic ring sign and the hyperdense interventricular septum and myocardium in detection of anaemia. The second purpose was an interest to determine whether the degree of tissue attenuation could accurately predict specific ranges of haemoglobin levels. 


\section{MATERIALS AND METHODS}

We performed a retrospective review on 70 patients who had undergone unenhanced CT of the thorax at the Govt. Medical College and SSG hospital, Vadodara between October 2016 and November 2016. No interaction was made with the patients. Necessary history and investigations were noted from the history sheet filled prior to the scan.

\section{Patients}

The patients had haemoglobin levels obtained within 72 hours of an unenhanced CT thorax. Haemoglobin was calculated using the standard hospital laboratory assay. Patients ranged in age of 18-80 years. Patients under the age of 18 years were excluded from the study. Anaemia was defined as a haemoglobin level below $14 \mathrm{~g} / \mathrm{dL}$ for men and below $12 \mathrm{~g} / \mathrm{dL}$ for women.

\section{CT Scan Analysis}

Patients were scanned in a 16 slice GE multidetector Computed Tomography scan machine using $1.2 \mathrm{~mm}$ thickness, 1.75 pitch, $200 \mathrm{~mA}$, and $120 \mathrm{kVp}$. Unenhanced CT images were evaluated on a preset mediastinal window (window width, $400 \mathrm{HU}$; window level, $40 \mathrm{HU}$ ). Each examination, assessed with attenuation (in HU) measured with a rectangular region of interest. Regions of interest were placed in the lumen of aorta at the region of bifurcation of Main Pulmonary Artery. Also, the relative hyperdense appearance of interventricular septum and myocardium and aortic ring sign was noted. All examinations were reported by the consultant radiologists of Government Medical College and Sri Sayajirao Gaekwad Hospital, Vadodara.

\section{Statistical Analysis}

The sensitivity, specificity, correlation and the $\mathrm{p}$ value were calculated and analysis was done.

\section{RESULTS}

Anaemia was defined as a haemoglobin level below $12 \mathrm{~g} / \mathrm{dL}$ for women and below $14 \mathrm{~g} / \mathrm{dL}$ for men Subjective image analysis revealed that the Aorta CT attenuation value was more sensitive and the interventricular septum sign was more specific. The aortic ring sign was negative in 8 out of 46 patients with anaemia. False positive aortic ring sign was seen in elderly patients with atherosclerotic wall calcifications. However, these patients did not present with any signs of anaemia nor was proved in laboratory analysis. The interventricular septum sign was highly specific since in our study we did not encounter any case of storage disorder. The Aorta CT attenuation value although faired reasonably was found to show false negative results in 7 out of 46 patients. This variable failed to detect cases of mild anaemia. However, a good correlation $(\mathrm{r}=0.63, \mathrm{p}<0.05)$ was observed between the aortic CT attenuation values and the haemoglobin levels in the study group.

\begin{tabular}{|c|c|c|}
\hline & Sensitivity & Specificity \\
\hline Aortic ring sign & $82 \%$ & $85 \%$ \\
\hline Interventricular septum sign & $69 \%$ & $100 \%$ \\
\hline $\begin{array}{c}\text { Aorta CT attenuation value } \\
(\leq 35 \mathrm{Hu})\end{array}$ & $85 \%$ & $94 \%$ \\
\hline
\end{tabular}

\section{DISCUSSION}

The prevalence of anaemia in a developing country like India is very alarming. Its prevalence is very high in patients with malignancy, chronic diseases, post-traumatic patients which is an economic burden because of increased hospital stay duration.(17) It is important to recognise this condition early since early diagnosis of this condition reduces the morbidity, hospital stay duration and aids in the better recovery of the patient.

The data obtained during our study is available from the routine CT examinations. No additional radiation dose is incurred. Modern day CT protocols involve the use of IV contrast in almost all cases unless contraindicated in patients with abnormal renal function tests. However, often plain scans are done before injecting the contrast. These unenhanced plain computed tomography scans were made use of in the diagnosis of the condition. In the present report, we highlight the most sensitive and specific radiological analysis for detecting anaemia on unenhanced CT scan of the thorax.

As evidenced in the previous studies, interventricular septum sign (Fig. 3) was seen to be the most specific sign; however, it revealed a sensitivity of $69 \%$.(18) In contrast, the aortic ring sign (Fig. 1) revealed much better sensitivity of $85 \%$. However, the aortic ring sign was found to be less specific. This can be explained by the differentiation in the composition of the interventricular septum and the aortic wall. The aorta is seen to be made up of highly attenuating collagen and fibrous tissue while the myocardium is made up of lesser attenuating muscle fibres which is composed mainly of collagen and elastic fibres. Lower specificity of aortic ring sign can be explained by the existence of faint calcification within pre-existing atherosclerotic mural plaques, which are known by their dense appearance upon unenhanced CT. (Fig. 2). The myocardium, by comparison, is primarily built of less attenuating muscle fibres. Other less frequent aortic abnormalities such as Takayasu's arteritis and intramural aortic haematoma may induce similar effects. Identifying any calcified atherosclerotic mural plaques can thus invalidate the diagnostic significance of the aortic ring sign, whereas the smooth, uniform, and homogeneous appearance, and the absence of periaortic fat infiltration may be used as reliable exclusion criteria for any potential inflammatory or other disorder of the aortic wall.(19-21) The conditions affecting the myocardium like infective (bacterial like tuberculosis, viral like adenovirus), inflammatory states (Rheumatic Heart Disease) and deposition disease that resulted in increase in the density of the myocardium gave false positive results. (22-23)

Results of previous studies also have demonstrated similar HU cut-off values for describing anaemia; however, recognising that there is often a zone of transition under which mild anaemia and normal haemoglobin levels are difficult to differentiate, a cut-off value of $\leq 35 \mathrm{HU}$ for aortic blood pool attenuation showed a favourable sensitivity and specificity of $85 \%$ and $95 \%$, respectively. The false negative values were obtained in the transition zone, in patients with mild anaemia. However, the aortic pool CT attenuation values is a useful adjunct to the diagnosis of anaemia.

The limitation of our study was probably due to the short sample size, due to the image quality resulting from the difference in the body mass index of patients undergoing scans. However, applying a weight-adapted scan protocol, 
image quality without significant noise contribution, $(24,25)$ has been previously shown to yield high and constant despite this limitation; analysing the $\mathrm{HU}$ value of the aortic blood pool can be considered as a useful adjunct that could resolve a wide range of diagnostic uncertainties.

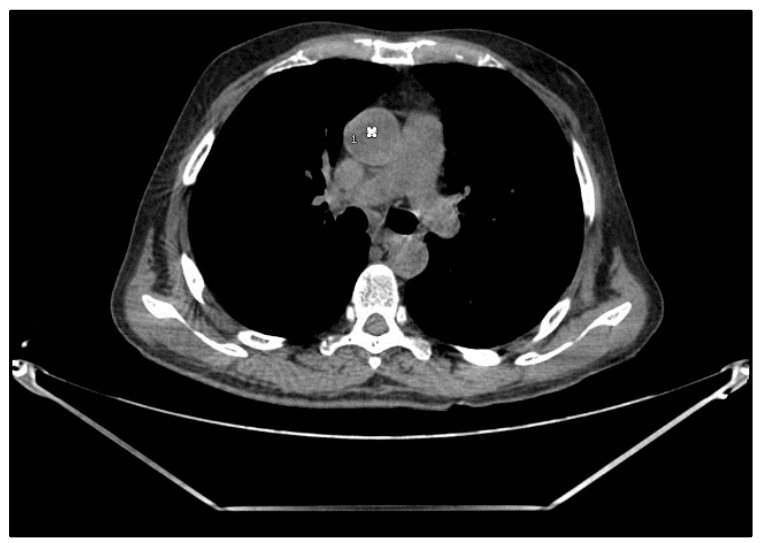

Figure 1. Aortic ring sign, shows the prominent aortic wall with intraluminal aortic $H U=20$ in a patient with proven anaemia in unenhanced CT thorax

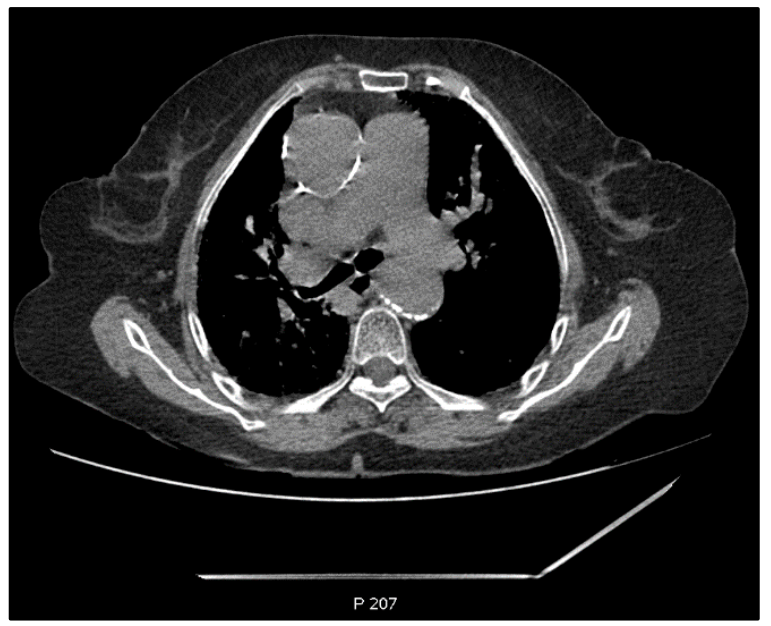

Figure 2. False aortic ring sign, shows the prominent aortic wall (age related calcification of aortic wall) in an elderly patient with normal haematological profile

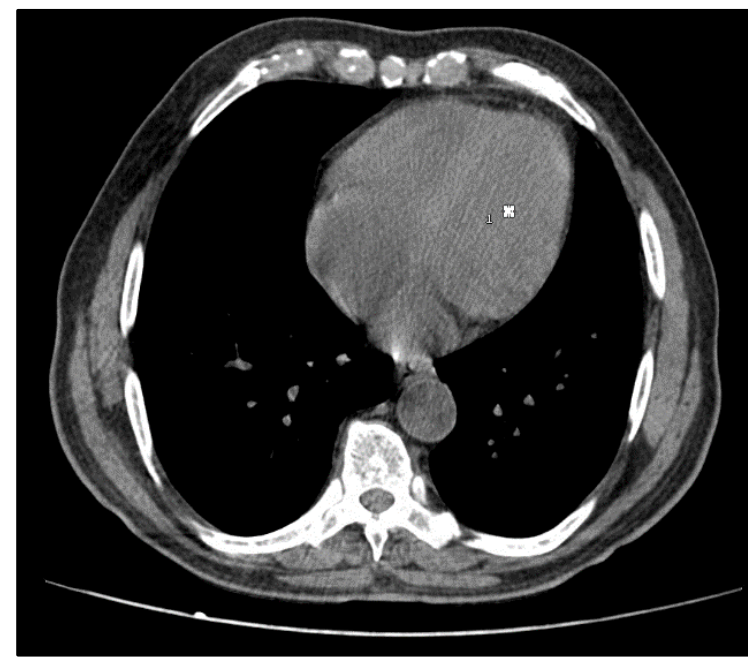

Figure 3. Interventricular septum sign, shows the prominent interventricular septum and myocardium in a patient with anaemia

\section{CONCLUSION}

The results demonstrate the significant correlation between the haematological haemoglobin assay and the aortic attenuation value. This provides a feasible means to detect anaemia in unenhanced CT examination, and we conclude that this readily available information may provide a valuable adjunct in the interpretation of such examinations.

Currently, the observed correlation is not strong enough to offer a definite diagnosis of anaemia; however, a larger sample size is likely to strengthen the predictive value of an HU cut-off value for anaemia. Such a cut-off value would provide a feasible means to assess anaemia when haematologic laboratory investigations are lacking.

\section{REFERENCES}

[1] New PF, Aronow S. Attenuation measurements of whole blood and blood fractions in computed tomography. Radiology 1976;121(3 Pt 1):635-40.

[2] Norman D, Price D, Boyd D, et al. Quantitative aspects of computed tomography of the blood and cerebrospinal fluid. Radiology 1977;123(2):335-8.

[3] Kamel EM, Rizzo E, Duchosal MA, et al. Radiological profile of anemia on enhanced MDCT of thorax. Eur Radiol 2008;18(9):1863-8.

[4] Groopman JE, Itri LM. Chemotherapy-induced anemia in adults: incidence and treatment. J Natl Cancer Inst 1999;91(19):1616-34.

[5] Spence RK. Medical and economic impact of anemia in hospitalized patients. Am J Health Syst Pharm 2007;64(16 Suppl 11):S3-10.

[6] Ania BJ, Suman VJ, Fairbanks VF, et al. Incidence of anemia in older people: an epidemiologic study in a well-defined population. J Am Geriatr Soc 1997;45(7):825-31.

[7] GBD 2013 Mortality and Causes of Death, Collaborators. Global, regional, and national age-sex specific all-cause and cause-specific mortality for 240 causes of death, 1990-2013: a systematic analysis for the Global Burden of Disease Study 2013. Lancet 2015;385(9963):117-71.

[8] Van de Wiel JC, Wang Y, Xu DM, et al. Neglectable benefit of searching for incidental findings in the Dutch-Belgian lung cancer screening trial (NELSON) using low-dose multidetector CT. Eur Radiol 2007;17(6):1474-82.

[9] Stillman AE, Oudkerk M, Ackerman M, et al. Use of multidetector computed tomography for the assessment of acute chest pain: a consensus statement of the North American society of cardiac imaging and the European society of cardiac radiology. Int J Cardiovasc Imaging 2007;23(4):415-27.

[10] Goetze S, Pannu HK, Wahl RL. Clinically significant abnormal findings on the "nondiagnostic" CT portion of low-amperage-CT attenuation-corrected myocardial perfusion SPECT/CT studies. J Nucl Med 2006;47(8):1312-8.

[11] Foster M, Nolan RL, Lam M. Prediction of anemia on unenhanced computed tomography of the thorax. Can Assoc Radiol J 2003;54(1):26-30.

[12] Shiotani S, Kohno M, Ohashi N, et al. Hyperattenuating aortic wall on post-mortem computed tomography (PMCT). Radiat Med 2002;20(4):201-6. 
[13] Sato Y, Kozawa E, Tsukuda S, et al. Feasibility of CT attenuation values in the diagnosis of anemia. Nippon Igaku Hoshasen Gakkai Zasshi 2004;64(7):394-7.

[14] Doppman JL, Rienmuller R, Lissner J. The visualized interventricular septum on cardiac computed tomography: a clue to the presence of severe anemia. J Comput Assist Tomogr 1981;5(2):157-60.

[15] Title RS, Harper K, Nelson E, et al. Observer performance in assessing anemia on thoracic CT. AJR Am J Roentgenol 2005;185(5):1240-4.

[16] Wojtowicz J, Rzymski K, Czarnecki R. Severe anaemia: its CT findings in the cardiovascular system. Eur J Radiol 1983;3(2):108-11.

[17] Corcoran HL, Cook DE, Proto AV. Diagnosis of anemia on computed tomography scans of the thorax. J Comput Tomogr 1988;12(2):116-21.

[18] Collins AJ, Gillespie S, Kelly BE. Can computed tomography identify patients with anaemia? Ulster Med J 2001;70(2):116-8.

[19] Sharma S, Sharma S, Taneja K, et al. Morphologic mural changes in the aorta revealed by CT in patients with nonspecific aortoarteritis (Takayasu's arteritis). AJR Am J Roentgenol 1996;167(5):1321-5.
[20] Chung JW, Park JH, Im JG, et al. Spiral CT angiography of the thoracic aorta. Radiographics 1996;16(4):81124.

[21] Castaner E, Andreu M, Gallardo X, et al. CT in nontraumatic acute thoracic aortic disease: typical and atypical features and complications. Radiographics 2003;23:S93-110.

[22] Cooper LT. Myocarditis. New Engl J Med 2009;360(15):1526-38.

[23] Kindermann I, Barth C, Mahfoud F, et al. Update on myocarditis. J Am Coll Cardiol 2012;59(9):779-92.

[24] Mulkens TH, Bellinck P, Baeyaert M, et al. Use of an automatic exposure control mechanism for dose optimization in multi-detector row CT examinations: clinical evaluation. Radiology 2005;237(1):213-23.

[25] Kemper J, Regier M, Bansmann PM, et al. Multidetector CT urography: experimental analysis of radiation dose reduction in an animal model. Eur Radiol 2007;17(9):2318-24. 